\title{
On swapping the states of two qudits
}

\author{
Colin M Wilmott \\ Institut für Theortische Physik III, Heinrich-Heine-Universität, Düsseldorf, \\ Germany \\ E-mail: wilmott@thphy.uni-duesseldorf.de
}

\begin{abstract}
The SWAP gate has become an integral feature of quantum circuit architectures and is designed to permute the states of two qubits through the use of the well-known controlled-NOT gate. We consider the question of whether a two-qudit quantum circuit composed entirely from instances of the generalised controlled-NOT gate can be constructed to permute the states of two qudits. Arguing via the signature of a permutation, we demonstrate the impossibility of such circuits for dimensions $d \equiv 3(\bmod 4)$.
\end{abstract}

PACS numbers: 02.20.Bb, 03.67.Lx

\section{Introduction}

A prerequisite for quantum computation is the successful implementation of multiplequbit quantum gates. The most elementary of all multiple-qubit quantum gates is premised by two-qubit controlled unitary operators, and a classic example is the controlled-NOT (CNOT) gate. The CNOT gate has been shown to provide a basis for a measurement set that permits the construction of syndrome tables used in error correction (Chuang \& Yamamoto 1997). Additionally, the CNOT gate has assumed a central role in the theory of quantum computation. It is the quantum mechanical analogue of the classical connective XOR gate and is a principle component in universal computation. Furthermore, Barenco et al. (1995) have shown that any multiplequbit quantum operation may be restricted to compositions of single-qubit gates and instances of the CNOT gate. It is for this reason that we say the quantum gate library consisting of single-qubit gates and the CNOT gate is universal. As a consequence, the CNOT gate has acquired the special status as the hallmark of multi-qubit control (Vidal \& Dawson 2004).

In recent years, researchers in universal quantum computation have done considerable work optimizing quantum circuit constructions. Vatan \& Williams (2004) constructed a quantum circuit for general two-qubit operations which requires fifteen single-qubit gates and at most three CNOT gates. A crucial aspect of this result is the demand that the SWAP gate requires at least three CNOT gates. The SWAP gate describes the cyclical permutation of the states of two qubits and has become an integral feature of the circuitry design for many quantum operators. It is a fundamental element in the circuit implementation of Shor's algorithm (Fowler et al. 2004), and Liang \& Li (2005) maintain that experimentally realising the SWAP gate is a necessary condition for the networkability of quantum computation. 
(a)

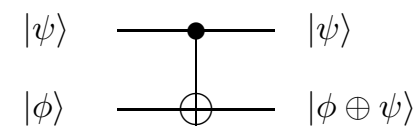

(b)

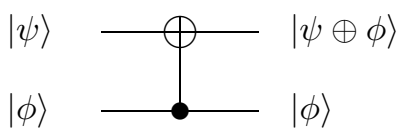

Figure 1. Circuit descriptions for the CNOT gate types. (a) The CNOT1 gate; the state of control system $|\psi\rangle \in \mathcal{H}_{\mathcal{A}}$ remains unchanged after application whereas the state of the target system $|\phi\rangle \in \mathcal{H}_{\mathcal{B}}$ is transformed under modular arithmetic to the state $|\phi \oplus \psi\rangle$. (b) The CNOT2 gate in which the roles of systems $\mathcal{H}_{\mathcal{A}}$ and $\mathcal{H}_{\mathcal{B}}$ are reversed.

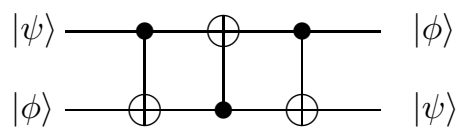

Figure 2. The SWAP gate illustrating the cyclical permutation of two qubits. System $\mathcal{A}$ begins in the state $|\psi\rangle$ and ends in the state $|\phi\rangle$ while system $\mathcal{B}$ begins in the state $|\phi\rangle$ and ends in the state $|\psi\rangle$.

In this paper, we examine the possibility of constructing a two-qudit SWAP gate using only instances of the generalised CNOT gate to permute the states of two qudits. Section 2 introduces preliminary material from the theory of permutations which will serve as a basis for our study. Section 3 considers the particular problem of whether a two-qutrit quantum circuit composed entirely from instances of the twoqutrit CNOT gate can be constructed to permute the states of two qutrits. Finally, section 4 generalises the results of section 3 to two-qudit quantum systems before demonstrating the impossibility of two-qudit SWAP gates using only instances of the generalised CNOT gate for dimensions $d \equiv 3(\bmod 4)$.

\section{Preliminaries}

\subsection{Elementary quantum gates}

Let $\mathcal{H}$ denote the $d$-dimensional complex Hilbert space $\mathbb{C}^{d}$. Fix each orthonormal basis state of the $d$-dimensional Hilbert space to correspond to an element of $\mathbb{Z}_{d}$; as such the basis $\{|0\rangle,|1\rangle, \ldots,|d-1\rangle\} \subset \mathbb{C}^{d}$ whose elements correspond to the column vectors of the identity matrix $\mathbb{I}_{d}$ is called the computational basis. A qudit is a $d$ dimensional quantum state $|\psi\rangle \in \mathcal{H}$ written as $|\psi\rangle=\sum_{i=0}^{d-1} \alpha_{i}|i\rangle$ where $\alpha_{i} \in \mathbb{C}$ and $\sum_{i=0}^{d-1}\left|\alpha_{i}\right|^{2}=1$. Given $d$-dimensional Hilbert spaces $\mathcal{H}_{\mathcal{A}}$ and $\mathcal{H}_{\mathcal{B}}$, consider the set of $d^{2} \times d^{2}$ unitary transformations $U \in U\left(d^{2}\right)$ that act on the two-qudit quantum system $\mathcal{H}_{\mathcal{A}} \otimes \mathcal{H}_{\mathcal{B}}$. Let $U_{\text {CNOT1 }} \in U\left(d^{2}\right)$ represent the generalised CNOT gate that has control qudit $|\psi\rangle \in \mathcal{H}_{\mathcal{A}}$ and target qudit $|\phi\rangle \in \mathcal{H}_{\mathcal{B}}$. The action of $U_{\mathrm{CNOT1}}$ on the set of basis 
states $|m\rangle \otimes|n\rangle$ of $\mathcal{H}_{\mathcal{A}} \otimes \mathcal{H}_{\mathcal{B}}$ is given by

$$
U_{\mathrm{CNOT1}}|m\rangle \otimes|n\rangle=|m\rangle \otimes|n \oplus m\rangle, \quad m, n \in \mathbb{Z}_{d},
$$

with $\oplus$ denoting addition modulo $d$. Similarly, let $U_{\text {СNот2 }} \in U\left(d^{2}\right)$ denote the generalised CNOT gate having control qudit $|\phi\rangle \in \mathcal{H}_{\mathcal{B}}$ and target qudit $|\psi\rangle \in \mathcal{H}_{\mathcal{A}}$. The action of $U_{\text {Спот2 }}$ on the set of basis states $|m\rangle \otimes|n\rangle$ of $\mathcal{H}_{\mathcal{A}} \otimes \mathcal{H}_{\mathcal{B}}$ is written

$$
U_{\mathrm{CNOT2}}|m\rangle \otimes|n\rangle=|m \oplus n\rangle \otimes|n\rangle, \quad m, n \in \mathbb{Z}_{d} .
$$

Figure 1 provides the quantum gate circuitry representation for the respective CNOT types while figure 2 illustrates the well-known SWAP gate for permuting the states of two qubits.

\subsection{Permutation groups}

Consider the set $\mathrm{N}=\{1,2, \ldots, n\}$ and let $\sigma: \mathrm{N} \mapsto \mathrm{N}$ be a bijection. Let $\sigma=\left[\begin{array}{cccc}1 & 2 & \ldots & n \\ i_{1} & i_{2} & \ldots & i_{n}\end{array}\right]$ be a permutation of the set $\mathrm{N}$ with $i_{k} \in \mathrm{N}$ denoting the image of $k \in \mathrm{N}$ under $\sigma$. Let $\sigma$ and $\tau$ be two permutations of $\mathrm{N}$. Define the product $\sigma \cdot \tau$ by $(\sigma \cdot \tau)(i)=\sigma(\tau(i)), i \in \mathrm{N}$, to be the composition of the mapping $\tau$ followed by $\sigma$. These permutations taken with (.) form the group $S_{n}$ called the symmetric group of degree $n$.

Given the permutation $\sigma \in S_{n}$ and for each $i \in \mathrm{N}$, let us consider the sequence $i, \sigma(i), \sigma^{2}(i), \ldots$. Since $\sigma$ is a bijection and $\mathrm{N}$ is finite there exist a smallest positive integer $\ell=\ell(i)$ depending on $i$ such that $\sigma^{\ell}(i)=i$. The orbit of $i$ under $\sigma$ then consists of the elements $i, \sigma(i), \ldots, \sigma^{\ell-1}(i)$. By a cycle of $\sigma$, we mean the ordered set $\left(i, \sigma(i), \ldots, \sigma^{\ell-1}(i)\right)$ which sends $i$ into $\sigma(i), \sigma(i)$ into $\sigma^{2}(i), \ldots, \sigma^{\ell-2}(i)$ into $\sigma^{\ell-1}(i)$, and $\sigma^{\ell-1}(i)$ into $i$ and leaves all other elements of $\mathrm{N}$ fixed. Such a cycle is called an $\ell$-cycle. We refer to 2-cycles as transpositions and note that any permutation can be written as a product of transpositions. A pair of elements $\{\sigma(i), \sigma(j)\}$ is an inversion in a permutation $\sigma$ if $i<j$ and $\sigma(i)>\sigma(j)$. The number of transpositions in any such product is even if and only if the number of inversions is even. Consequently, we say such a permutation is even. A similar case holds for odd permutations.

Lemma 2.1 Every permutation can be uniquely expressed as a product of disjoint cycles.

Proof. Let $\sigma$ be a permutation. Then the cycles of the permutation are of the form $i, \sigma(i), \ldots, \sigma^{\ell-1}(i)$. Since the cycles are disjoint and by the multiplication of cycles, we have it that the image of $i \in N$ under $\sigma$ is the same as the image under the product, $\varsigma$, of all the disjoint cycles of $\sigma$. Then $\sigma$ and $\varsigma$ have the same effect on every element in $N$, hence, $\sigma=\varsigma$.

Every permutation $\sigma \in S_{n}$ has a cycle decomposition that is unique up to the ordering of the cycles and up to a cyclic permutation of the elements within each cycle. Further, if $\sigma \in S_{n}$ and $\sigma$ is written as the product of disjoint cycles of length $n_{1}, \ldots, n_{k}$, with $n_{i} \leq n_{i+1}$, we say $\left(n_{1}, \ldots, n_{k}\right)$ is the cycle type of $\sigma$. As a result of Lemma 2.1, every permutation can be written as a product of transpositions. Since the number of transpositions needed to represent a given permutation is either even or odd, we define the signature of a permutation as

$$
\operatorname{sgn}(\sigma)= \begin{cases}+1 & \text { if } \sigma \text { is even } \\ -1 & \text { if } \sigma \text { is odd }\end{cases}
$$


To each permutation $\sigma \in S_{n}$, let us consider the corresponding permutation matrix $A_{\sigma}$ whereby

$$
A_{\sigma}(j, i)= \begin{cases}1 & \text { if } \sigma(i)=j \\ 0 & \text { otherwise. }\end{cases}
$$

The mapping $f: S_{n} \mapsto \operatorname{det}\left(A_{\sigma}\right)$ where

$$
\operatorname{det}\left(A_{\sigma}\right)=\sum_{\sigma \in S_{n}} \operatorname{sgn}(\sigma) \prod_{i=1}^{n} A_{\sigma(i), i}
$$

is a group homomorphism. The kernel of this homomorphism, $\operatorname{ker} f$, is the set of even permutations. Consequently, we have it that $\sigma \in S_{n}$ is even if and only if $\operatorname{det}\left(A_{\sigma}\right)$ equals +1 .

\section{On swapping the states of two qutrits}

Let $d=3$ and consider the following problem. Given a pair of qutrit quantum systems, system $\mathcal{A}$ in the state $|\psi\rangle$ and system $\mathcal{B}$ in the state $|\phi\rangle$, and using only instances of the two-qutrit CNOT gate, determine if it is possible permute the states of the corresponding systems so that system $\mathcal{A}$ ends in the state $|\phi\rangle$ while system $\mathcal{B}$ ends in the state $|\psi\rangle$.

Problem 3.1 Given qutrits $|\psi\rangle \in \mathcal{H}_{\mathcal{A}}$ and $|\phi\rangle \in \mathcal{H}_{\mathcal{B}}$ and using only instances of the two-qutrit CNOT gate, determine if it is possible to construct a two-qutrit quantum circuit that permutes the states of the quantum systems $\mathcal{H}_{\mathcal{A}}$ and $\mathcal{H}_{\mathcal{B}}$ such that $|\psi\rangle \otimes|\phi\rangle \in \mathcal{H}_{\mathcal{A}} \otimes \mathcal{H}_{\mathcal{B}}$ is mapped to $|\phi\rangle \otimes|\psi\rangle \in \mathcal{H}_{\mathcal{A}} \otimes \mathcal{H}_{\mathcal{B}}$.

We now show that for a pair of qutrits, it is not possible to permute the states $|\psi\rangle \otimes|\phi\rangle \in \mathcal{H}_{\mathcal{A}} \otimes \mathcal{H}_{\mathcal{B}}$ using only instances of the two-qutrit CNOT gate.

Any two-qutrit quantum circuit composed entirely from instances of the twoqutrit CNOT gate can be written in terms of the two-qutrit CNOT1 and CNOT2 gates. The action of the two-qutrit CNOT1 gate on the basis states $|m\rangle \otimes|n\rangle \in$ $\mathcal{H}_{\mathcal{A}} \otimes \mathcal{H}_{\mathcal{B}}, m, n \in \mathbb{Z}_{3}$, is described by the unitary transformation $U_{\mathrm{CNOT1}} \in U(9)$ given by

$$
U_{\mathrm{CNOT1}}|m\rangle \otimes|n\rangle=|m\rangle \otimes|n \oplus m\rangle, \quad m, n \in \mathbb{Z}_{3},
$$

where $\oplus$ denotes addition modulo 3. Figure 3 (a) provides the matrix description for the two-qutrit CNOT1 gate. A similar description for the two-qutrit CNOT2 gate holds, and figure 3 (b) provides the corresponding matrix description. Note also that the two-qutrit CNOT1 and CNOT2 gates can be described in the following way. The permutation matrix corresponding to the two-qutrit CNOT1 gate takes the value 1 in row $3 m+n$ and column $3 m+(n \ominus m), m, n \in \mathbb{Z}_{3}$. Similarly, the matrix corresponding to the two-qutrit CNOT2 gate takes the value 1 in row $3 m+n$ column $3(m \ominus n)+n$, $m, n \in \mathbb{Z}_{3}$. Importantly, both of these matrix descriptions have determinant +1 as the permutations corresponding to their the respective matrices are even.

Let us now assume there exists a two-qutrit quantum circuit composed entirely from instances of the two-qutrit CNOT gate types which permutes the states of two qutrits. By assumption, such a circuit will then be a composition of two-qutrit CNOT1 and CNOT2 gates. It then follows that any composition of two-qutrit CNOT1 and CNOT2 gates will be equivalent to some product of their respective unitary matrix descriptions. Such a matrix product will necessarily have determinant +1 as both 
(a) $\left(\begin{array}{lllllllll}1 & 0 & 0 & 0 & 0 & 0 & 0 & 0 & 0 \\ 0 & 1 & 0 & 0 & 0 & 0 & 0 & 0 & 0 \\ 0 & 0 & 1 & 0 & 0 & 0 & 0 & 0 & 0 \\ 0 & 0 & 0 & 0 & 0 & 1 & 0 & 0 & 0 \\ 0 & 0 & 0 & 1 & 0 & 0 & 0 & 0 & 0 \\ 0 & 0 & 0 & 0 & 1 & 0 & 0 & 0 & 0 \\ 0 & 0 & 0 & 0 & 0 & 0 & 0 & 1 & 0 \\ 0 & 0 & 0 & 0 & 0 & 0 & 0 & 0 & 1 \\ 0 & 0 & 0 & 0 & 0 & 0 & 1 & 0 & 0\end{array}\right)$

(b) $\left(\begin{array}{lllllllll}1 & 0 & 0 & 0 & 0 & 0 & 0 & 0 & 0 \\ 0 & 0 & 0 & 0 & 0 & 0 & 0 & 1 & 0 \\ 0 & 0 & 0 & 0 & 0 & 1 & 0 & 0 & 0 \\ 0 & 0 & 0 & 1 & 0 & 0 & 0 & 0 & 0 \\ 0 & 1 & 0 & 0 & 0 & 0 & 0 & 0 & 0 \\ 0 & 0 & 0 & 0 & 0 & 0 & 0 & 0 & 1 \\ 0 & 0 & 0 & 0 & 0 & 0 & 1 & 0 & 0 \\ 0 & 0 & 0 & 0 & 1 & 0 & 0 & 0 & 0 \\ 0 & 0 & 1 & 0 & 0 & 0 & 0 & 0 & 0\end{array}\right)$

Figure 3. Matrix representations of two-qutrit CNOT types. (a) The matrix representation for the two-qutrit CNOT1 gate. (b) The matrix description for the two-qutrit CNOT2 gate.

$$
\left(\begin{array}{lllllllll}
1 & 0 & 0 & 0 & 0 & 0 & 0 & 0 & 0 \\
0 & 0 & 0 & 1 & 0 & 0 & 0 & 0 & 0 \\
0 & 0 & 0 & 0 & 0 & 0 & 1 & 0 & 0 \\
0 & 1 & 0 & 0 & 0 & 0 & 0 & 0 & 0 \\
0 & 0 & 0 & 0 & 1 & 0 & 0 & 0 & 0 \\
0 & 0 & 0 & 0 & 0 & 0 & 0 & 1 & 0 \\
0 & 0 & 1 & 0 & 0 & 0 & 0 & 0 & 0 \\
0 & 0 & 0 & 0 & 0 & 1 & 0 & 0 & 0 \\
0 & 0 & 0 & 0 & 0 & 0 & 0 & 0 & 1
\end{array}\right)
$$

Figure 4. The matrix $U^{*} \in U(9)$ that permutes the states of two qutrits.

constituent elements have determinant +1 . However, figure 4 represents the unitary transformation $U^{*} \in U(9)$ required to permute the states of two qutrits. Such a swap matrix takes the value 1 in row $3 m+n$ column $3 n+m$, and has determinant -1 . Therefore, no composition of two-qutrit CNOT gate types can yield the required matrix, and the result follows. 


\section{On swapping the states of two qudits}

We generalize problem 3.1 to higher dimensional quantum systems and ask if it is possible to construct a two-qudit quantum circuit composed entirely from instances of the generalised CNOT gate to permute the states of two qudit quantum systems.

Problem 4.1 Given a pair of qudits $|\psi\rangle \in \mathcal{H}_{\mathcal{A}}$ and $|\phi\rangle \in \mathcal{H}_{\mathcal{B}}$ and using only instances of the generalised CNOT gate, determine if it is possible to construct a two-qudit quantum circuit to permute the state $|\psi\rangle \otimes|\phi\rangle \in \mathcal{H}_{\mathcal{A}} \otimes \mathcal{H}_{\mathcal{B}}$.

We have shown in section 3 that the unitary matrices corresponding to the twoqutrit CNOT1 and CNOT2 gate types both have determinant +1 , and this contrasted significantly with the determinant of the unitary matrix required to permute the states of a pair of qutrits. Consequently, no composition of the former could yield the latter and the result followed. There is, however, another way to look the problem of permuting the states of two quantum systems using only instances of the generalised CNOT gate, and it is the following. Firstly, in examining the qutrit case, we note that the permutations corresponding to the two-qutrit CNOT1 matrix and the swap matrix $U^{*} \in U(9)$ are

$$
\begin{aligned}
\sigma_{\mathrm{CNOT1}} & =\left[\begin{array}{lllllllll}
0 & 1 & 2 & 3 & 4 & 5 & 6 & 7 & 8 \\
0 & 1 & 2 & 5 & 3 & 4 & 7 & 8 & 6
\end{array}\right] \\
\sigma_{U^{*}} & =\left[\begin{array}{lllllllll}
0 & 1 & 2 & 3 & 4 & 5 & 6 & 7 & 8 \\
0 & 5 & 6 & 7 & 4 & 1 & 2 & 3 & 8
\end{array}\right]
\end{aligned}
$$

respectively. In particular, these permutations describe, respectively, the action of both the two-qutrit CNOT1 gate and the swap matrix $U^{*}$ on the set of basis states $|m\rangle \otimes|n\rangle \in \mathcal{H}_{\mathcal{A}} \otimes \mathcal{H}_{\mathcal{B}}, m, n \in \mathbb{Z}_{3}$. The cycle type for two-qutrit CNOT gate is $(1,1,1,3,3)$ while the cycle type for the swap matrix $U^{*}$ is $(1,1,1,2,2,2)$. Hence, the two-qutrit CNOT gate fixes three basis states and permutes the remaining states in two cycles of length 3. Each such cycle may be written as a product of two transpositions. Whence, the signature of the two-qutrit CNOT permutation is +1 . On the other hand, the permutation describing swap of a pair of qutrit states contains three fixed elements and a set of three transpositions and therefore the signature of this permutation is -1 . Consequently, it follows that within a two-qutrit quantum circuit architecture, no composition of the two-qutrit CNOT gate types alone can permute the states of two qutrits.

More generally, the two-qudit CNOT gate that acts on a pair of $d$-dimensional quantum systems corresponds to a permutation of the $d^{2}$ basis states. For prime dimensions $d=p$, the permutation associated with the generalised CNOT1 gate fixes $d$ basis states and induces $(d-1)$ cycles of length $d$, each of which may be written as a product of $(d-1)$ transpositions. The generalised CNOT1 gate permutation is then a composition of $(d-1)^{2}$ basis state transpositions. A similar case holds for the generalised CNOT2 gate in that corresponding mapping fixes $d$ basis elements induces $(d-1)$ cycles where each is a product of $(d-1)$ transpositions. Therefore, the signature of the generalised CNOT permutation is -1 for dimension $d=2$ and +1 for odd prime dimensions.

Now, let us consider the unitary matrix $U^{*} \in U\left(d^{2}\right)$ that swaps the states of two qudits. This matrix permutes the basis states of a pair of qudits thereby mapping the two-qudit state $|\psi\rangle \otimes|\phi\rangle \in \mathcal{H}_{\mathcal{A}} \otimes \mathcal{H}_{\mathcal{B}}$ to the state $|\phi\rangle \otimes|\psi\rangle \in \mathcal{H}_{\mathcal{A}} \otimes \mathcal{H}_{\mathcal{B}}$. Such a transformation corresponds to a permutation of the $d^{2}$ basis states $|m\rangle \otimes|n\rangle \in$ 
$\mathcal{H}_{\mathcal{A}} \otimes \mathcal{H}_{\mathcal{B}}, m, n \in \mathbb{Z}_{d}$. Under this mapping, there are $d$ fixed basis elements and $d(d-1) / 2$ transpositions on all remaining basis states. Consequently, the signature of the permutation describing the two-qudit SWAP gate is -1 for dimensions $d \equiv 2$ or $3(\bmod 4)$ and +1 for dimensions $d \equiv 0$ or $1(\bmod 4)$. It then follows that two-qudit quantum circuits composed entirely from instances of the generalised CNOT gate can not permute the states of two qudits when $d \equiv 3(\bmod 4)$.

\section{Conclusion}

We considered the problem of constructing a two-qudit SWAP gate using only instances of the generalized CNOT gate. We discussed the idea of signature for a permutation and identified, via this signature, when it is possible to permute the states of two qudits using only instances of the generalised CNOT gate. Based on this argument, we demonstrated the impossibility of constructing a two-qudit SWAP gate using only instances of the generalised CNOT gate for dimensions $d \equiv 3(\bmod 4)$. This may be of interest the for more general task of constructing a $k$-qudit SWAP gate using only generalised CNOTs. Finally, our understanding of the symmetric group and its decompositions may well be enhanced by considering how to design quantum circuits to realise certain subgroups of this group.

\section{Acknowledgments}

The author wishes to thank Prof. Peter Wild for many helpful suggestions.

\section{References}

Barenco A, Bennett C H, Cleve R, DiVincenzo D P, Margolus N, Shor P, Sleator T, Smolin J A \& Weinfurter H 1995 Phys. Rev. A 52(5), 3457-3467.

Chuang I L \& Yamamoto Y 1997 Phys. Rev. A 55(1), 114-127.

Fowler A G, Devitt S J \& Hollenberg L C L 2004 Quant. Info. Comput. 4, 237.

Liang L \& Li C 2005 Phys. Rev. A 72(2), 024303.

Vatan F \& Williams C 2004 Phys. Rev. A 69(3), 032315.

Vidal G \& Dawson C M 2004 Phys. Rev. A 69(1), 010301. 